\title{
Civilisations
}

Revue internationale d'anthropologie et de sciences

humaines

61-1| 2012

Au-delà du consensus patrimonial

\section{Digestion patrimoniale}

Contestations autour d'un ancien musée des colonies à Paris

Anne Monjaret et Mélanie Roustan

\section{(2) OpenEdition}

\section{Journals}

Édition électronique

URL : http://journals.openedition.org/civilisations/3123

DOI : $10.4000 /$ civilisations.3123

ISSN : 2032-0442

Éditeur

Institut de sociologie de l'Université Libre de Bruxelles

\section{Édition imprimée}

Date de publication : 22 décembre 2012

Pagination : 23-42

ISBN : 2-87263-038-4

ISSN : 0009-8140

\section{Référence électronique}

Anne Monjaret et Mélanie Roustan, « Digestion patrimoniale », Civilisations [En ligne], 61-1 | 2012, mis en ligne le 30 décembre 2015, consulté le 20 avril 2019. URL : http://journals.openedition.org/ civilisations/3123; DOI : 10.4000/civilisations.3123

(c) Tous droits réservés 


\title{
Digestion patrimoniale \\ Contestations autour d'un ancien musée des colonies à Paris
}

\author{
Anne MONJARET et Mélanie ROUSTAN
}

\begin{abstract}
Résumé : Si le Palais de la Porte Dorée, à Paris, a toujours abrité un musée, ses contenus et leur interprétation ont varié : colonies, outre-mer, arts lointains puis immigration. Ces évolutions patrimoniales n'ont pas été sans heurts, que la requalification porte sur les collections ou le bâtiment, construit en 1931 pour l'Exposition coloniale. Certaines contestations visent les destinations patrimoniales de l'édifice : grèves des personnels, pour la fermeture du Musée national des arts d'Afrique et d'Océanie (2003) ; manifestations de militants extérieurs, controverses et démissions d'intellectuels, lors de l'installation de la Cité nationale de l'Histoire de l'immigration (2007). D'autres contestations ne prennent plus ce patrimoine pour enjeu mais comme levier pour servir une cause : indépendance d'anciennes colonies ; régularisation d'immigrés "sans-papiers" ".'occupation des lieux par des "travailleurs grévistes sans-papiers » durant l'hiver 2010 est au centre de l'attention, à travers l'analyse de l'originalité d'outils de contestation relevant de la sphère patrimoniale : accompagnement de la lutte par des photographes ou illustrateurs, édition d'ouvrages, montage d'expositions par des militants, collecte en continu par le musée... Est ici interrogée la capacité quasi immédiate de «digestion » des événements présents par le paradigme patrimonial.
\end{abstract}

Mots-clés : musée d'ethnologie, colonies, immigration, patrimoine, mouvements sociaux.

\begin{abstract}
The Palais de la Porte Dorée in Paris has always been a museum, but its collections and their interpretation varied trough time: colonies, overseas, remote arts and finally immigration. These patrimonial changes and requalification provoked conflicts focused either on the collections or the building built in 1931 for the colonial exhibition. Contests relates to the patrimonial use of the building: strikes of employees in 2003 when the National Museum of Arts from Africa and Oceania closed; demonstrations of outside activists; controversies and resignations of intellectuals in 2007 when the Palais became the National City of the History of Immigration. Other contests use heritage not as an issue but as a lever to defend a cause: the independence of former colonies or the regularisation of illegal immigrants with a particular focus on the sit-in by illegal "worker strikers" during winter 2010. The authors try to grasp the originality of contestation tools belonging to the heritage area - support by photographers and illustrators, publication of books, exhibitions created by activists or permanent collection by the museum - and to tackle the nearly immediate capacity to "digest" of present events via the heritage paradigm.
\end{abstract}

Keywords: museum of ethnology, colonies, immigration, heritage, social movements. 
L

orsque nous arrivons au Palais de la Porte Dorée le mardi $1^{\text {er }}$ février 2011 vers 18 heures, pour le vernissage de l'exposition Zoom Cieslewicz montée par le Musée national de l'histoire et des cultures de l'immigration à Paris, le bâtiment est entouré de fourgons de police. Dans la nuit froide de cette fin d'hiver parisien, les forces de l'ordre patrouillent autour du musée et sont postées à la grille d'entrée. Elles filtrent les arrivants qui doivent montrer leur carton d'invitation en guise de sésame. Un peu impressionnées par ce déploiement, nous pénétrons à l'intérieur de l'édifice. Là, tout semble paisible et normal. Nous sommes accueillies par les agents d'accueil et de surveillance et les équipes de la conservation, puis grimpons les escaliers pour accéder à l'exposition. L'espace est empli d'une foule dense. Anonymes, représentants du monde des arts, professionnels des musées et journalistes se pressent autour des œuvres de ce graphiste polonais de renom, en attendant la visite officielle de Jacques Toubon, président du Conseil d'orientation de la Cité nationale de l'histoire de l'immigration (CNHI). Lorsque nous saluons le directeur de l'établissement et devant notre étonnement face à la situation, il nous explique, un peu confus, que vendredi 28 janvier matin, la police a bloqué l'accès du bâtiment, empêchant, manu militari, le collectif de " travailleurs grévistes sans-papiers » d'y pénétrer, sur ordre de la Préfecture de Police. Depuis bientôt quatre mois, y était installé un collectif de militants issus pour la plupart d'Afrique de l'Ouest. Ces derniers réclamaient leur régularisation sur le territoire français, via l'occupation d'un lieu symbolique : la CNHI, établie dans un palais destiné initialement au Musée des Colonies.

Nous proposons dans cet article $^{1}$ de décrypter cet événement et sa genèse, à la lumière d'une longue tradition de controverses, de contestations autour de ce palais construit à l'occasion de l'exposition coloniale internationale de 1931. Ainsi nous nous situons entre une ethnographie institutionnelle et une anthropologie des usages sociaux et politiques du lieu. Quelles résistances aux réinterprétations patrimoniales y observe-t-on ? Comment la valorisation patrimoniale se voit-elle utilisée comme levier de revendications? Au fil des mutations du Palais de la Porte Dorée, emblématique du discours idéologique de la nation française, nous étudions ces multiples tensions et les manières dont les objets et rhétoriques du patrimoine sont mobilisés pour articuler le passé et le présent, l'ailleurs et l'ici, l'autre et le même. Nous analysons la capacité du paradigme patrimonial à « digérer » quasi immédiatement ses mouvements de contestations.

\section{Contester la patrimonialisation et ses évolutions}

Si le Palais de la Porte Dorée a toujours abrité un musée, ses contenus et leur interprétation ont varié : colonies, outre-mer, arts lointains et enfin immigration. Ces évolutions patrimoniales n’ont pas été sans heurts, que leurs enjeux portent sur la

1 Les résultats présentés ici se fondent sur divers travaux menés depuis la fin des années 1990 au Palais de la Porte Dorée, à Paris : enquêtes de réception auprès des publics, ethnographie de l'institution au début des années 2000 (avec Jacqueline Eidelman), poursuivie en tandem dix ans plus tard dans le cadre d'une recherche subventionnée par le ministère français de la Culture et de la Communication. 
requalification des collections ou sur celle du bâtiment. Les contestations révèlent une préoccupation quant aux mutations du musée et au destin de ses équipes, autant qu'une inquiétude sur les usages du Palais et de ses symboles. Pour la période récente, elles prennent la forme de grèves des personnels, à l'époque de la fermeture du Musée national des arts d'Afrique et d'Océanie (MNAAO) en 2003, de manifestations de militants extérieurs, de controverses d'intellectuels, voire de démissions, lors de l'installation de la CNHI en 2007.

\section{Illustration 1}

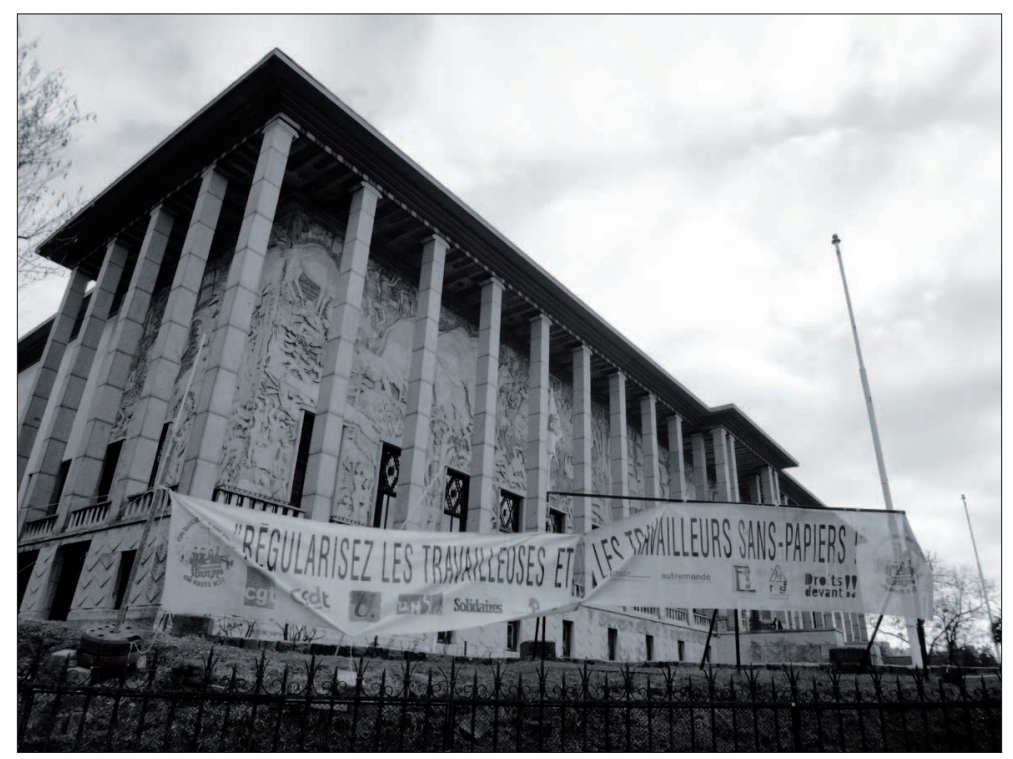

Banderoles autour de la Cité nationale de l'histoire de l'immigration. (C) Mélanie Roustan, janvier 2011.

Faire la grève

Les actions de contestation ont toujours fait partie du paysage du MNAAO, comme dans bien des lieux de travail. Toutefois certaines périodes ont été plus intenses que d'autres. En 2001, le projet de « loi sur les musées » provoque un tollé car elle remet en cause « le principe de l'inaliénabilité des collections publiques ». Un Mouvement de défense des musées (ou Comité Patrimoine et Résistance) est créé sous l'égide de chercheurs, conservateurs, archéologues, etc., soutenu par l'intersyndicale des musées. Ses revendications dépassent la question de l'avenir des musées pour recouvrir la sphère de la fonction publique, aux prises avec les nouveaux impératifs 
d'externalisation². Les musées d'ethnologie sont concernés : «Nous vous demandons en outre d'interdire le déménagement, la dispersion des collections du musée de l'Homme, du MNAAO, du MNATP ${ }^{3}$ et la destruction des institutions qui les abritent», mentionne l'un des documents diffusés. La demande est catégorique. Mais si les personnels du musée de l'Homme sont fortement mobilisés, apparaissant souvent sur le devant de la scène médiatique (Grognet 2009), ceux du MNAAO semblent plus fatalistes et ne suivent pas le mouvement avec autant de ferveur. Quelquesuns participent toutefois aux actions. L'ensemble du personnel vit difficilement le sort qui lui est réservé, comme tous les travailleurs touchés par la fermeture d'un établissement (Linhart et al. 2004 ; Monjaret 2005). Première hypothèse, en tant qu'agents d'État, ils ont conscience de la sécurité de l'emploi dont ils bénéficient, ce qui les freinerait à se plaindre et, a fortiori, à manifester ouvertement. Dix ans plus tard, le représentant CGT que nous avions alors rencontré, actuellement en poste au musée des Civilisations de l'Europe et de la Méditerranée (MuCEM), s'explique mieux l'attitude de ses collègues du MNAAO à l'époque : l'accompagnement pour le reclassement auquel chacun des agents a bénéficié les aurait rendus moins combatifs. La prise en charge individuelle par l'institution du devenir des personnels serait parvenue à un retrait vers la sphère privée qui conduit à délaisser la participation à des luttes collectives ${ }^{4}$. Passé le choc de l'annonce de la fermeture, le travail reprend et en 2002, le MNAAO nous apparaît socialement ensommeillé. Autre hypothèse, une enquête menée auprès du personnel et commandée par la direction du musée, donna lieu à un bel ouvrage et à une exposition temporaire sur les souvenirs de ceux qui ont travaillé dans l'établissement, intitulés «MAAO Mémoires » (Eidelman et al. 2002). Cela aurait eu pour effet de neutraliser une partie des tensions. Le processus de patrimonialisation « en direct » insiste sur la reconnaissance et demeure tourné vers le passé (Monjaret et al. 2005). Les personnels, qu'ils soient amenés à quitter ou non les lieux, restent soucieux de leur avenir et de celui du bâtiment. Des pronostics se font sur la nature du futur établissement qui y sera hébergé. Mais l'état des débats n'a rien de commun avec l'ébullition intellectuelle suscitée en 2004 par le projet, très controversé, d'y installer la CNHI.

\section{Débattre et démissionner}

L'implantation de la CNHI au Palais de la Porte Dorée n'est pas allée de soi. Le bâtiment a, depuis son origine, été mis en cause par des minorités militantes. À

2 Notons également que sur le site du comité, les arguments développés sur les nouvelles orientations marchandes du champ culturel - « rêve du commerçant », « Il faut faire directement de Léonard de Vinci un employé de Disneyland »- ne sont finalement pas si éloignés de ceux avancés en 1931 par le groupe d'intellectuels, célèbres surréalistes dont André Breton, qui signa le Manifeste « Ne visitez pas l'exposition Coloniale », s'érigeant contre cette manifestation « Luna Park de Vincennes » et dénonçant les méfaits politiques bourgeois du colonialisme.

3 Musée national des Arts et traditions populaires (MNATP), aujourd'hui Musée des Civilisations de l'Europe et de la Méditerranée (MuCEM).

4 Il reste que le 26 février 2001, l'appel à la grève commune avec le Musée de l'Homme est largement entendu, preuve d'une certaine inquiétude et ce, même si cette contestation n'aura pas d'effets flagrants sur la marche des choses. 
l'époque de sa construction, son destin se confond avec celui de l'Exposition coloniale internationale de 1931. Édifice construit pour durer, en tant que Musée permanent des Colonies, il prend place à la lisière du bois de Vincennes, à Paris. Pensé comme un « temple » à la gloire des colonies, il fait office de centre d'apparat, au milieu des multiples reproductions d'architectures exotiques mises en scène comme autant de « merveilles » de l'Empire français, animées par des « indigènes » amenés sur place pour l'occasion. Le succès de l'Exposition est considérable. Toutefois, quelques voix se font déjà entendre pour protester contre cet événement : un groupe de surréalistes, mené par André Breton, enjoint les publics à le boycotter, au nom des valeurs morales d'égalité ; s'y opposent de même le Parti communiste français et la CGTU5, ainsi que quelques associations, par exemple d'intellectuels sénégalais. Des manifestations sont organisées, des tracts distribués, des centaines d'affichettes collées sur les arbres de Paris... Le mouvement contestataire existe, mais il semble infime comparé aux millions de visiteurs qui se pressent à l'Exposition. Lorsque celle-ci se termine, tous les édifices provisoires sont démontés, et, après quelques années de travaux, rouvrent le Parc zoologique du bois de Vincennes et le Palais des Colonies - ce dernier en tant que Musée des Colonies et de la France extérieure (1933). Il devient rapidement Musée de la France d'Outre-mer (1935), et avec les indépendances, change d'orientation, et se transforme en Musée des arts africains et océaniens (1960) puis en Musée national des arts d'Afrique et d'Océanie (1990). En 2003, il doit fermer ses portes car ses collections rejoignent le Musée du quai Branly en construction. Le bâtiment, vide, cherche preneur. Le débat autour de sa reconversion fait rage au sein du milieu des musées et des instances chargées de la Culture : doit-il être considéré comme un témoin architectural, joyau du style Art Déco, ou comme un témoin historique, archétype de l'idéologie coloniale du début du $20^{\mathrm{e}}$ siècle ? Si sa valeur patrimoniale n'est pas remise en cause, quel doit en être l'axe majeur? Histoire de l'architecture et des arts mobiliers ou Histoire tout court ?

Il devra finalement accueillir un établissement portant sur l'histoire de l'immigration. L'installation de la future CNHI dans un tel lieu ne paraît pas évidente. L'institution, fruit de l'agrégation progressive d'associations luttant pour la connaissance de l'histoire et la reconnaissance des mémoires de l'immigration en France (Hommes et migrations 2004 ; Diasporas 2005), met presque deux décennies à devenir un projet porté par l'État (El Yazami et Schwartz 2001), destiné à la valorisation d'un «patrimoine des migrants »(Museum International 2007). Elle peine à trouver son implantation. Au-delà du classique débat entre l'architecture contemporaine et le patrimoine bâti, entre la construction ad hoc et la réutilisation de l'existant, la controverse dépasse le champ architectural pour se déplacer vers le politique. La discussion porte d'emblée sur la pertinence d'installer un musée

5 La Confédération générale du travail unitaire (CGTU) était un syndicat français, proche du Parti communiste français, mais réunissant également des tendances révolutionnaire et libertaire, ayant existé entre 1921 et 1936.

6 À ce titre, il est intéressant d'observer les évolutions de sa fiche de classement à l'Inventaire supplémentaire des monuments historiques (liste officielle des bâtiments et sites protégés au titre de leur valeur historique et patrimoniale par l'État français, et bénéficiant ainsi d'une protection particulière). 
dédié à la mise en patrimoine de l'immigration (en constituant une collection nationale) dans un édifice marqué du sceau de l'idéologie coloniale du siècle passé (Blanc-Chaléard 2006 ; Bancel et Blanchard 2007 ; Cohen 2007 ; Green 2011). La symbolique d'un monument est-elle réversible ? Peut-on ramener le phénomène migratoire à la problématique coloniale ? La « dette » coloniale est-elle soluble dans la légitimation culturelle de l'immigration, via la notion de patrimoine ? La décision d'une implantation dans l'ancien Musée des Colonies, ravive les discussions autour de son destin, que d'aucuns auraient voulu voir se transformer en un lieu de contremémoire, un « musée du musée » (Bancel et Blanchard op. cit.).

La dimension politique du projet de la CNHI a toujours été explicite. L'idée de « changer le regard de la société française sur l'immigration » fait partie des objectifs donnés par l'État à la mission de préfiguration du projet (Toubon 2004 : 51). Mais lorsque Nicolas Sarkozy arrive au pouvoir en mai 2007, son annonce de la création d'un grand ministère de l'Immigration, de l'Intégration, de l'Identité nationale et du Développement solidaire est perçue comme le signe d'un virage à droite de l'État. À quelques mois de l'ouverture de l'établissement, le risque d'amalgame entre ces différents messages contradictoires est jugé trop grand par certains membres de son comité scientifique. Huit d'entre eux démissionnent, sept historiens et un démographe. Parmi eux, Gérard Noiriel, qui était parvenu en 2005, avec la création d'un Comité de vigilance face aux usages publics de l'histoire ${ }^{7}$, à faire abroger un projet de loi prônant l'enseignement du « rôle positif » de la colonisation. Interviewé par le quotidien Libération $^{8}$, il explique :

Ce label associant immigration et identité nationale charrie des représentations négatives. Désormais, tout le monde va prononcer quotidiennement le nom de ce ministère, et ce qui auparavant ne s'entendait que dans la bouche des gens d'extrême droite va être complètement banalisé.

Le renouvellement du contexte politique tend à donner une toute autre tonalité à la CNHI, qui se retrouve en porte-à-faux par rapport à son message initial.

\section{Manifester}

C'est donc dans un contexte politique particulièrement tendu que la CNHI ouvre ses portes au public. Les forces de l'ordre présentes aux grilles de l'établissement constituent un signe des risques de dérapage supposés et permettent de mesurer à quel point la polémique n'est pas retombée. En ce mercredi 10 octobre 2007, l'ouverture de la CNHI ressemble pourtant par son silence officiel à la fermeture du MNAAO. Les membres du gouvernement ne s'y rendent pas ; seul le maire de Paris, Bertrand Delanoë, y assiste. L'inauguration se transforme en non-événement (Bensa, Fassin 2002). « Pas de flonflons pour la Cité de l'immigration », peut-on lire dans la presse (Libération 10/10/2007). Paradoxalement, cette situation cristallise les critiques ayant trait à la CNHI, la rendant visible aux yeux de ceux qui s'y intéressent : « La Cité de 
l'immigration ouvre sur fond de polémique » (Reuters 10/10/2007) ; « La Cité de l'immigration naît dans la douleur » (Le Figaro 08/10/07).

\section{Illustration 2}

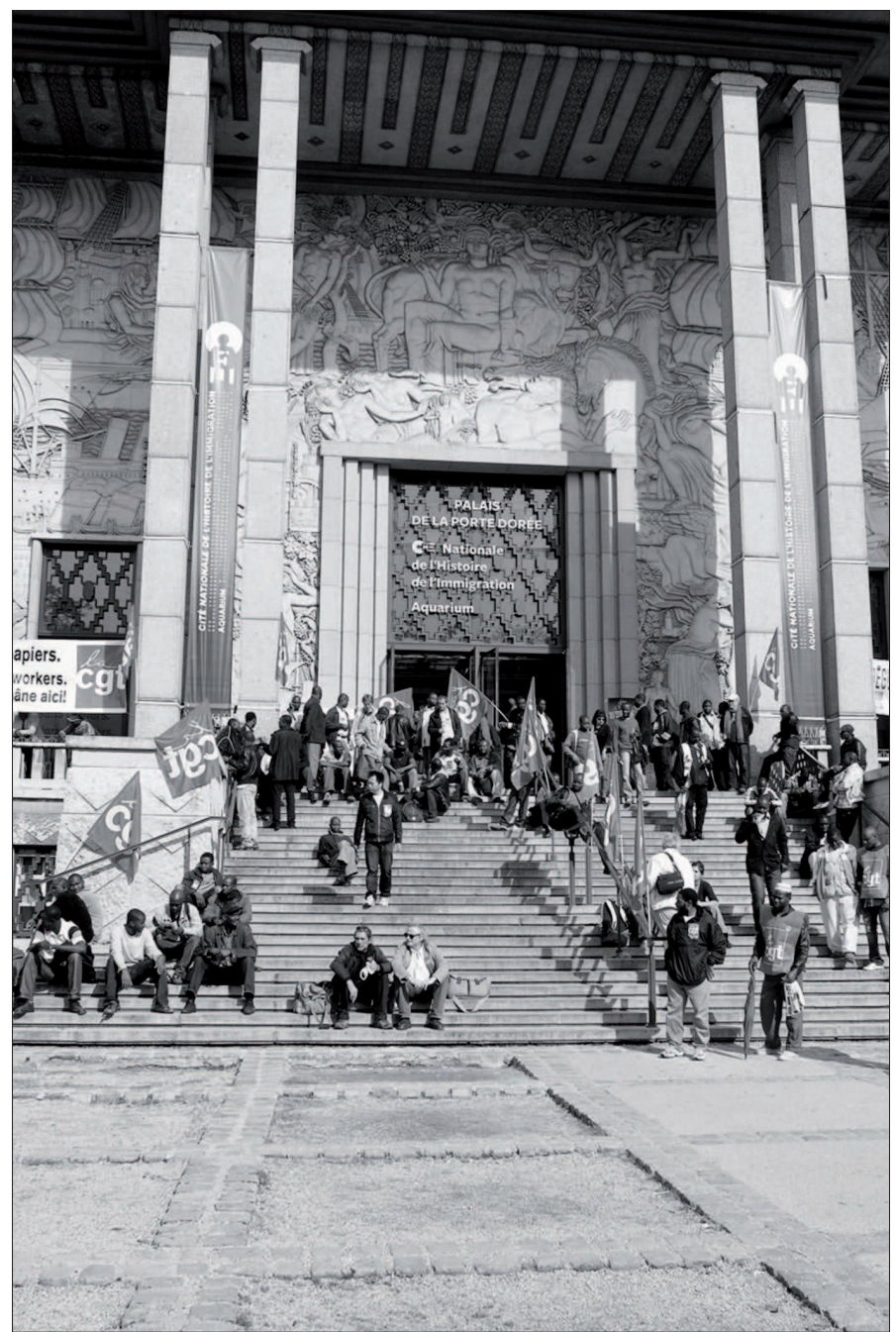

Les « travailleurs grévistes sans-papiers » occupent la Cité nationale de l'histoire de l'immigration.

(C) Bruno Combes, octobre 2010.

Si l'inauguration se fait sans les ministres de tutelle, elle prend pour certains les formes d'une action citoyenne. Devant le Palais de la Porte Dorée, en dehors du périmètre de l'établissement bloqué par la police, des associations (dont la Ligue des droits de l'Homme) et des représentants des historiens démissionnaires ont improvisé leur inauguration sur la chaussée, au su et au vu des passants, des participants et 
des journalistes couvrant l'affaire. Comme le veut la tradition, un ruban est coupé, puis des discours sont prononcés par différents partenaires qui montent sur le pupitre dressé pour l'occasion. Ils entendent affirmer leur position en matière d'immigration'. « En l'absence d'inauguration officielle, la ligue des droits de l'homme et d'autres associations appellent ce matin à une "inauguration citoyenne de la Cité" " (Libération 10/10/2007). Au non-événement, répond donc un contre-événement.

Le sort s'acharne sur la CNHI. Le 30 mars 2009, jour de l'inauguration de la médiathèque Abdelmalek Sayad ${ }^{10}$, qui fait figure d'inauguration officielle, la cérémonie est perturbée. Prévoyant les affrontements, la ministre de la Culture, Christine Albanel et la ministre de l'Enseignement supérieur, Valerie Pécresse, renoncent à s'y rendre. Les forces de l'ordre quadrillent le secteur et les invités doivent montrer patte blanche pour pénétrer dans les lieux. Les collectifs des sans-papiers présents, sont tenus dehors à l'écart. Pourtant, alors que la visite de la médiathèque s'achève et que les officiels s'apprêtent à donner leur discours, des manifestants réussissent à entrer dans les locaux - et parmi eux, des " étudiants » et des "intermittents du spectacle 》et commencent, d'après les témoignages ${ }^{11}$, à scander des slogans contre la politique gouvernementale. Ces actions militantes sont suffisamment fortes pour dissuader les deux autres ministres présents de rester : l'un s'éclipse rapidement et quitte la CNHI, quand l'autre reste mais s'isole dans une pièce avec des journalistes pour s'expliquer. Cette inauguration, somme toute "gâchée », laisse à ses témoins institutionnels, aujourd'hui encore, un goût amer, l'impression d'une incompréhension. L'événement est en quelque sorte tué par le contre-événement.

Lors de ces deux célébrations officielles, événement et contre-événement se répondent. Mais ce constat n'est pas propre aux inaugurations, les manifestations associées à la fermeture d'établissements professionnels combinent généralement l'officiel et l'officieux, l'institutionnel et l'opposition (Monjaret 2008). En dehors des témoignages, il nous reste pour mesurer l'impact de ces deux moments phares, les dossiers de presse compilés par le personnel du service de la médiathèque, soucieux de constituer à chaud un corpus, témoins, mémoires des lieux. Comme toujours, le Palais ne peut se soustraire à son histoire, entre colonie et immigration. Il sert de toile de fond à l'énonciation de convictions, continuellement sujettes à discussion. Il se dresse aussi comme l'étendard des causes sociales, il est brandi pour afficher toutes sortes de revendications.

\section{Utiliser le patrimoine pour contester, revendiquer}

Certaines contestations ne portent pas sur la destination patrimoniale du Palais mais s'en saisissent pour alerter l'opinion publique, faire passer un message, servir une cause : indépendance des anciennes colonies, régularisation des « travailleurs immigrés sans-papiers ». Sous des formes plus ou moins pacifiques, ces actions

9 Pour des photographies et une vidéo de l'inauguration citoyenne de la CNHI, voir sur le site du collectif12: [www.collectif12.com/spip.php?article21]

10 Du nom du sociologue franco-algérien.

11 Voir aussi à ce sujet : « La Police inaugure la Cité de l'immigration », Libération 31/03/2009. 
utilisent l'efficacité symbolique des lieux pour interroger l'idée contemporaine de la nation française et en discuter les principes. Les édifices sont parfois mis à mal. Une dégradation qui vaut contestation du message qu'ils incarnent. Ils sont alors touchés dans ce qu'ils représentent, littéralement. D'autres fois, ils se retrouvent occupés. Les militants entendent faire un usage instrumental de l'institution. L'occupation n'a pas visée d'appropriation mais de revendication - le terme « occuper » introduisant « une nuance guerrière qui marque bien le caractère conflictuel des usages du bâtiment » (Fabre 2009 : 30). Il arrive que ces actions soient mises en mémoire et en culture simultanément à leur développement - posant la question de l'efficacité politique de la patrimonialisation « en temps réel ».

\section{Vandaliser}

Des actes terroristes visent à toucher les symboles du colonialisme. En 1983, le monument situé en face du Palais, dédié à la mission Congo-Nil, est l'objet d'un plastiquage. Lors de nos entretiens auprès des personnels du musée, les mémoires se font vagues quant au motif des activistes : "Ils revendiquaient un truc pour les Antilles », "C'était pour l'indépendance des Antilles 》, "Quelque chose à propos des Caraïbes ». Le guide du Musée daté de 1987 revient sur différents épisodes concernant ce monument : "Ce symbole d'une expédition héroïque a subi bien des avatars ; à peine construit, il s'effondrait, dut être bâché pendant l'Exposition Coloniale puis rebâti grâce à une souscription nationale. En 1983, il fut endommagé par un attentat dirigé contre son message jugé colonialiste. Restauré, il est désormais amputé de son principal acteur, le Commandant Marchand, pulvérisé par l'explosion ».

Si le Palais de la Porte Dorée n'est pas la cible directe de cette action, la déflagration de la bombe ne l'épargne pas : les vitres de façade volent en éclat. Un agent de surveillance, présent au moment des faits, se souvient : "Les vitres du musée en avaient souffert aussi. Mais ce n'était pas le musée qui était visé ». Une autre se remémore ce mois d'août où elle a été appelée en urgence : "C'était en face mais le musée a eu toute la façade abîmée, toutes les vitres soufflées... Heureusement c'était le soir, on avait déjà fermé. (...) Et puis on est resté fermé trois mois ensuite, ça a été réparé vite ». D'autres se rappellent avoir, durant plusieurs jours, ramassé le verre brisé «par grandes pelletées ».

Ce n'est ni la première ni la dernière fois que ce monument est visé. Lors de notre enquête en novembre 2001, nous apprenons de la bouche d'un agent : "Le monument à la mémoire du Commandant Marchand (...) vient d'être restauré d'ailleurs. Parce qu'il a été restauré, puis tagué, puis maintenant nettoyé, et puis dans quelques jours il sera de nouveau tagué ». En 2008, « Mayotte n'est pas à vendre, Mayotte est comorienne ", pouvait-on y lire. Ce tag est une réponse critique à la consultation engagée auprès des habitants de l'île de Mayotte pour son intégration à la liste des départements français (le vote se fera en faveur de cette départementalisation).

Parfois, les militants, ne se satisfaisant plus de l'espace public pour crier leurs mécontentements, choisissent de pénétrer de hauts lieux pour tenter d'être plus persuasifs et d'obtenir gain de cause : le Palais de la Porte Dorée appartient à cette catégorie de bâtiments assaillis, du moins occupés. 


\section{Illustration 3}

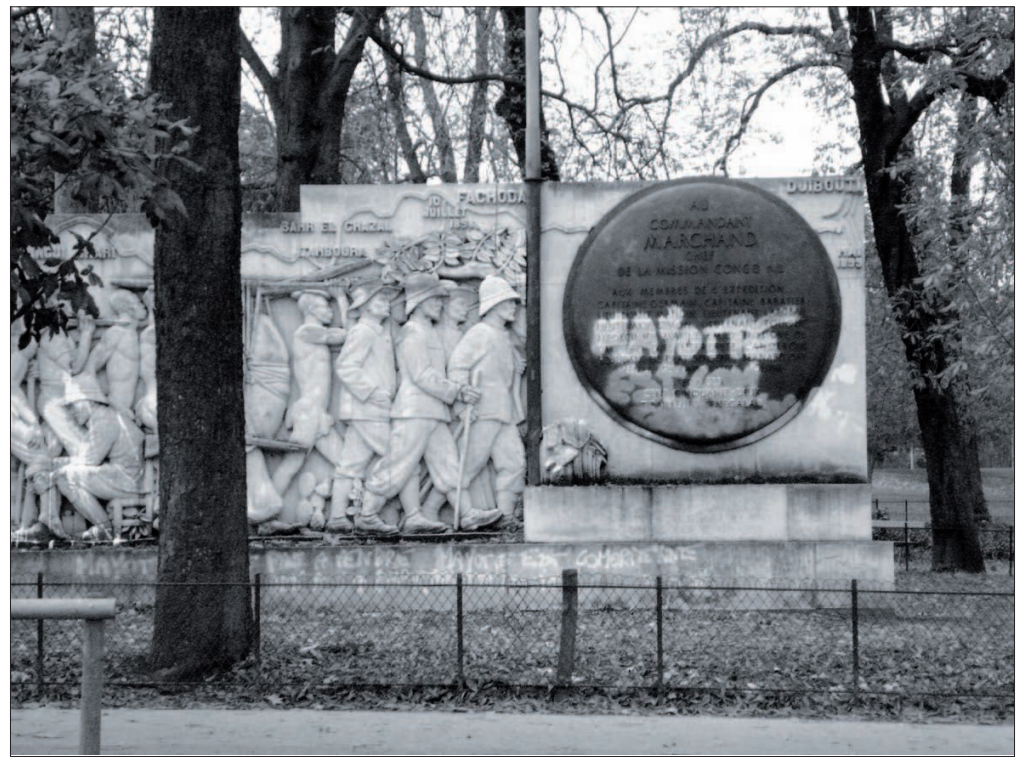

Graffiti politique concernant l'île de Mayotte,

sur le monument dit «du Commandant Marchand», en face du Palais de la Porte Dorée.

(C) Mélanie Roustan, octobre 2008 .

\section{Occuper}

Il est ponctuellement investi par des militants. Les témoignages recueillis ne s'accordent pas sur les dates et les profils des occupants (intermittents du spectacle, étudiants en archéologie, militants de l'association Droit Au Logement, travailleurs sans-papiers...), mais tous conviennent qu'il s'agit généralement d'une occupation d'une brève durée, une demi-journée tout au plus. L'occupation des lieux durant quatre mois à l'hiver 2010-2011, par un collectif de « travailleurs grévistes sans-papiers », se présente comme un épisode exceptionnel ${ }^{12}$.

Un précédent revient cependant à l'esprit des personnels rencontrés. Près de 15 jours avant le premier tour des élections présidentielles, le 5 avril 2002 après-midi exactement, alors que Karine Sapora, chorégraphe, prépare son spectacle au sein du MNAAO, des sans-papiers et leurs soutiens, militants associatifs et syndicaux, font irruption dans le hall du musée, s’y cantonnant par respect des œuvres, du public et des personnels, explique-t-on dans un communiqué du syndicat Sud Culture. Les forces de l'ordre, entrées par l'arrière du bâtiment, procèdent à l'expulsion des manifestants qui se rendront ensuite avec Monseigneur Gaillot, connu pour son

12 Notre ethnographie s'appuie ici sur les témoignages recueillis après-coup auprès des personnels de l'établissement et de quelques participants au mouvement, ainsi que sur l'analyse des médias (presse écrite et internet). 
soutien aux causes sociales, auprès du directeur de campagne de Jacques Chirac pour faire part de leurs revendications. Pour les militants, le choix du Palais n'est pas neutre : «Dans ce lieu hautement symbolique rappelant les profits colossaux de la colonisation, les enfants, les petits-enfants des colonisés d'hier exigent de MM Jospin et Chirac leur régularisation $»^{13}$. Le collectif Droits devant!! reprend dans son tract le titre du Manifeste de 1931 « Ne visitez pas l'exposition coloniale ! », et affiche un mot d'ordre révélateur « Colonisés hier au sud, esclaves aujourd'hui au nord », quand Sud Culture titre son communiqué « Colonisés hier, exploités aujourd'hui », comme pour rappeler avec insistance les méfaits des politiques de la France impérialiste. En 2007, les collectifs de sans-papiers seraient repassés dans les locaux. Puis le 30 mars 2009, lors de l'inauguration de la médiathèque de la CNHI, ils reviennent au Palais. Le MNAAO en 2002 puis la CNHI en 2009 et en 2010 : le bâtiment reste le symbole du couple colonisé/immigré. que les militants s'approprient.

En 2010 donc, le collectif des sans-papiers ${ }^{14}$, après avoir investi les marches de l'Opéra Bastille, se rend sur les marches du Palais de la Porte Dorée : deux symboles de l'histoire de la France pour clamer haut et fort que l'État ne doit pas oublier son engagement du 18 juin 2010 concernant leur régularisation (Barron et al. 2011 : 282). La CNHI, ce temple dédié aujourd'hui à l'histoire des immigrés, leur histoire, est en quelque sorte leur Palais : "parce que c'est l'immigration », mais aussi parce que c'est "leurs racines ». C'est également de leur point de vue une « antenne du ministère de l'Immigration » où ils peuvent trouver de l'aide afin d'obtenir les titres de séjour qui leur font défaut ou du moins l'octroi d'un récépissé de trois mois avec une autorisation de travail en attente de l'obtention du titre de séjour. " "Conformément à l'accord du 18 juin dernier signé avec le ministère de l'Immigration, nous avons déposé 1800 dossiers pendant l'été. Jusqu'à présent, seulement 58 cas ont été réglés et ont donné lieu à des autorisations de séjour provisoire", rappelle Sacko Fousseni, 28 ans, l'un des porte-parole du mouvement, qui a le soutien de onze syndicats » (Télérama, Sortir 20/10/2010). Il reste que pour certains observateurs, le choix de ce lieu tend à souligner davantage la figure de l'immigré que celle du travailleur. Ainsi, le 7 octobre 2010, près de trois ans après l'inauguration de la CNHI, comme pour célébrer son anniversaire, 500 sans-papiers, majoritairement des hommes originaires d'Afrique de l'Ouest, équipés de leurs sacs de couchage, franchissent, soutenus par la CGT et de nombreuses associations, les portes du Palais. Les médias couvrent l'événement peu habituel. La direction de la CNHI obtempère et accepte dans un premier temps le principe de l'occupation, jour et nuit. La « salle des fêtes » (le « forum »), cœur de la cité, et les espaces extérieurs sont investis. Très vite la direction demande aux sans-papiers de s'installer dans une salle latérale, pour ne pas perturber les activités de la Cité et ne pas gêner les visites du public. Puis en décembre, après d'âpres négociations, il est décidé que l'occupation se réduirait à la journée. Des locaux réservés aux activités culturelles et pédagogiques sont mis à disposition des grévistes pour poursuivre l'accompagnement et aider aux démarches administratives. Ces

13 Site Droits devant !! : [www.droitsdevant.org/article.php3?id_article=64] (consulté 12/10/2009)

14 Les mouvements de sans-papiers trouvent leur genèse en 1973, mais le collectif, tel qu'on le connaît aujourd'hui, prend forme en 2006 (Barron et al. 2011). 
« ateliers » deviennent l'« Ambassade des Travailleurs Sans-Papiers » (communiqué CGT Culture). Le 28 janvier 2011, il est mis fin au mouvement. Un des témoins raconte de façon condensée les étapes spatiales de ces quatre mois d'occupation :

Le début de l'occupation de jour dans la salle des fresques, ils ont commencé à avoir les récépissés, c'est là où on a commencé à faire des photos avec l'arrivée des récépissés. Après ils ont été déplacés, la CGT a demandé de quitter la salle pour celle d'à côté, et vu ce qui s'était passé à la Bastille, ils étaient quand même pas très chauds. Ils étaient d'abord déplacés d'une pièce à l'autre et après on leur a demandé de ne pas rester la nuit. Et ils ont été déplacés dans le couloir des ateliers et là ça avait chauffé, je me souviens de ce jour-là, ça avait vraiment chauffé, j'avais l'impression que ça allait dégénérer.

Durant cette période d'occupation, c'est dans une ambiance parfois tendue, parfois bon enfant, que s'organise le quotidien des grévistes. Certains d'entre eux sont préposés aux contrôles des allers et venues et assurent la sécurité dans leurs rangs. D'autres le sont au ménage, tentant de minimiser les dégradations, en particulier des sanitaires. D'autres encore le sont à la cuisine. Une petite " caisse » est mise à disposition pour les soutenir, mais la CGT leur fait aussi livrer des provisions et des repas :

Oui ils mangeaient par terre, il y a des gens qui venaient avec un fourgon leur apporter à manger le midi et le soir, il y avait des sacs poubelles partout dans le jardin ,

nous raconte un agent de surveillance. Un petit commerce s'instaure également,

À partir de $17 \mathrm{~h}$ ils venaient et ils restaient jusqu'à $21 \mathrm{~h}$, ils ne couchaient plus sur place mais ils faisaient du business. De bouffe essentiellement, il y en a qui vendaient des beignets sucrés, d'autres des oufs durs, d'autres des boissons (...).

Les premiers temps, des couchages sont installés dans la salle des fêtes : «Parce que c'est le lieu symbolique ». Un agent de la CNHI raconte :

Il m'a montré où sont ses origines dans la grande salle, en me disant sur les fresques "C'est là ! ». C'est pour ça qu'il a dormi dans la salle. (...) Il m'a dit "C'est là qu'il y a des choses qui m'appartiennent ", j'ai dit que ça appartenait à tout le monde, il m'a dit "Non, c'est pour ça qu'on ne part pas ».

Mais en décembre, l'occupation est limitée à la journée, des lieux sont alors vidés des matelas, déposés en tas sur le trottoir face au bâtiment. La vie se poursuit.

Pour passer le temps, les hommes palabrent, allongés sur des paillasses, ils jouent aux cartes, au baby-foot - empruntant ainsi un accessoire de l'exposition « Football et immigration ». Ils visitent le musée (l'exposition permanente leur est rendue gratuite) et ce qu'ils y trouvent entre en résonance avec leurs propres souvenirs :

Et il y a un travailleur gréviste qui a dit : " vous voyez ils sont venus chez moi il y a 10 ans dans mon village et ils ont pris cette photo ». Et il se reconnaissait. Et donc ils se sont dit ça nous appartient parce que tout ce qui est dans ce bâtiment, ça fait partie de nos vies,

nous rapporte le responsable des ressources humaines. Ils vont consulter les fonds de la médiathèque et se plonger dans un retour aux sources visuel. Ils se regroupent 
autour d'un musicien ou partagent le thé, rituel convivial qui ponctue la journée. Certains prient dans la salle des fêtes, d'autres aux abords du bâtiment.

\section{Illustration 4}

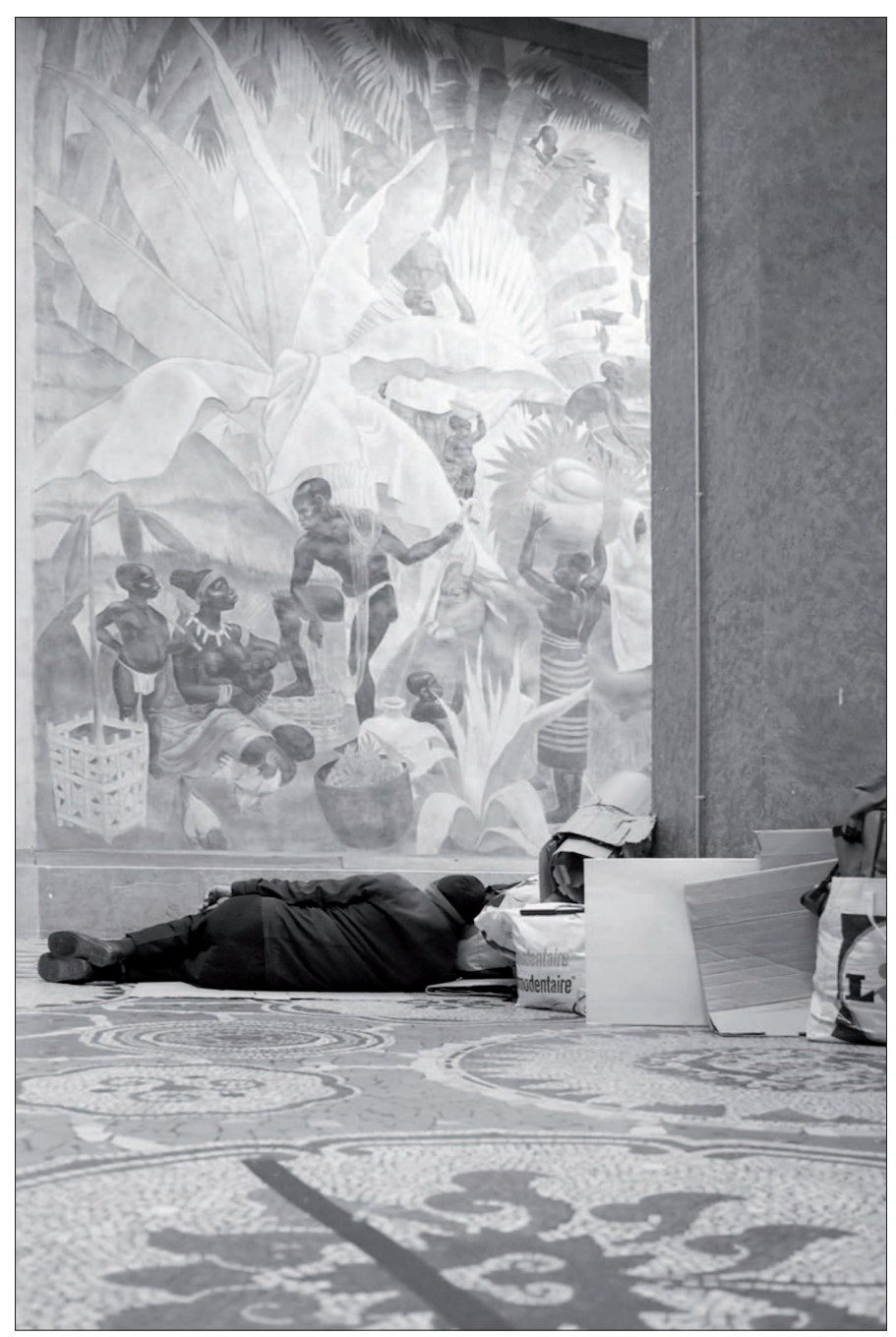

Moment de repos dans la grande salle centrale du Palais.

(C) Bruno Combes, novembre 2010. 


\section{Illustration 5}

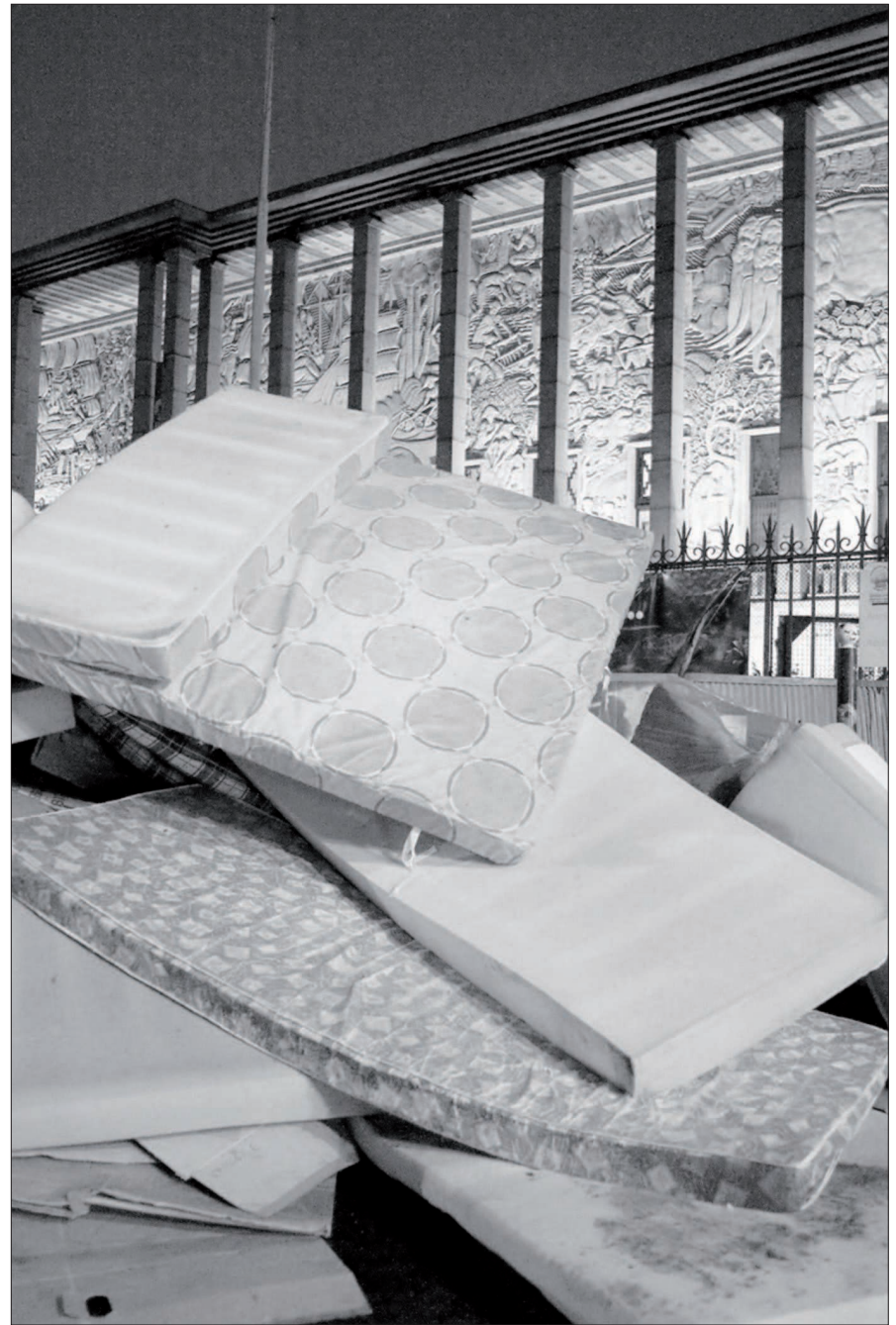

L'occupation de jour comme de nuit s'achève, les matelas sont immédiatement déposés sur le trottoir.

(C) Bruno Combes, décembre 2010.

En dehors de ces moments d'entre-soi, des séances d'accueil administratif et des rassemblements plus politiques rappellent les motifs de l'occupation. Les grévistes reçoivent des marques de sympathie de nombreux militants qui, parfois en famille, viennent leur renouveler leur soutien (Droits devant!! Ligue des droits de l'homme, 
Comité de soutien du $12^{\text {eme }}$ arrondissement ${ }^{15}$, etc.). Des représentantes de Femmes Egalité se seraient relayées de jour comme de nuit. Plus ponctuellement, des personnalités politiques leur rendent visite : Olivier Besancenot, leader du Nouveau Parti anticapitaliste (NPA) ou Cécile Duflot, alors secrétaire nationale des Verts (parti écologiste). Trois des huit historiens démissionnaires sont, de même, venus les saluer ${ }^{16}$.

Le représentant de la CGT veille au bon déroulement des opérations. Avec le leader du groupe des sans-papiers, décrit comme un homme charismatique sachant désamorcer les crises, ils temporisent les tensions. Les personnels de la CNHI ont dû faire face à la situation. Ils s'étonnent d'abord que les contestataires ne soient pas « évacués » par la police, comme à l'accoutumée. Leurs réactions sont diverses. Certains adoptent une position d'évitement, entrant et sortant par les escaliers de secours à l'arrière du bâtiment et travaillant comme si de rien n'était. D'autres, tenus par leur fonction, n'ont pas cette alternative : les membres de la direction assument leurs astreintes, les agents de surveillance en font leur quotidien, restant parfois la nuit, pour les plus haut placés dans la hiérarchie.

La direction nous a dit de gérer l'ouverture au public. Donc là, les quêtes, les files d'attentes aux toilettes, les visiteurs qui ne comprennent pas... La misère humaine aussi, les gens qui racontent leur vie... Les agents ont morflé : on n'est pas préparés à ça, on n'a pas le recul. Et puis de fil en aiguille, on s'est retrouvés tout seul, parce que les autres services se sont terrés dans leur bureaux, plus personne ne descendait dans le hall d'entrée et on s'est retrouvés tout seul et on a fini par travailler avec eux,

explique un agent d'encadrement. Les conditions de travail sont difficiles. Les surveillants retiennent le désordre, le froid : "Ils rentraient et ils sortaient, c'était pénible surtout à cause des portes ouvertes avec le froid, on avait les pieds gelés, c'était la folie ». Ils retiennent aussi le bruit, l'état déplorable des sanitaires, les visiteurs peu conciliants voire racistes, les fermetures sporadiques qui troublent le planning, et surtout la fatigue qui s'installe les mois durant - « ils étaient très affaiblis moralement ", confirme un responsable. Toutefois, ils ne se plaignent pas de la situation avec virulence, mesurant le drame social des sans-papiers dont ils n'envient pas le sort, et auquel, parfois, ils comparent leur destin plus heureux. Certains restent en retrait ou expriment quelques critiques, d'autres expliquent que cette expérience les a nourris mémorablement. Plus que de la sympathie, se construisent des relations d'empathie et d'amitié, permises grâce aux échanges journaliers. Cette attitude leur est parfois reprochée par leur hiérarchie qui considère que des agents d'État doivent savoir garder la distance qu'implique leur statut. La direction de la CNHI a dû faire face à une crise plutôt complexe, dont la longueur a fini par la mettre dans l'embarras. Si elle se confronte à un coût humain, le coût financier n'est pas négligeable. Les premiers mois, elle joue la conciliation, informe, via son site internet, le public du contexte, propose la gratuité des visites guidées le week-end. Elle souhaite éviter

15 Pour plus d'informations sur les formes de son implication dans le cadre de cette occupation, voir le site : [http://collectif12.org/]

16 [http://immigration.blogs.liberation.fr/coroller/2010/10/pour-sarko-lhistoire-de-limmigration-nefait-pas-partie-de-lhistoire-de-france.html] 
que son image soit ternie par l'événement ou que la fréquentation baisse. Puis oscillant entre ouverture et fermeture, la situation devient intenable : se profile bientôt l'inauguration de deux expositions attendues : Zoom Cieslewicz dont l'ouverture au public est prévue le 2 février 2011, et Polonia, le 2 mars. Le 28 janvier 2011, au matin, la police accueille les grévistes devant les grilles et leur bloque l'accès de la Cité. «Les forces de l'ordre comme seule réponse ! », s'exclame un communiqué du syndicat CGT Culture. Les associations qui soutiennent le mouvement prennent aussi le relais sur leurs sites internet, pour informer de la situation. Quant aux personnels, certains sont soulagés tandis que d'autres sont révoltés :

Après, ça s'est terminé par la décision de la préfecture de police : on encercle le bâtiment. Là, ça a été dur à vivre, parce qu'on était habitués à eux, on se demandait ce qu'ils allaient devenir, on était déjà habitués avec eux. On a été aussi très touchés parce que le soir de l'occupation, un des sans-papiers m'a appelé pour me remercier en me disant "je te remercie, je sais que tu as tout fait pour que ça se passe bien, tu remercieras toute l'équipe, on n'a jamais été reçus comme ça". Le mec, il était derrière le cordon de police, il avait pas ses papiers. Il vient nous voir de temps en temps, il nous appelle, on reste en contact. (...) Nous ce qui nous tient à cæur, c'est que les sans-papiers nous appellent pour nous remercier mais pas la direction. (...) on aurait dî aller jusqu'au bout de la négociation, on avait entamé ça, c'était pas un processus facile, il fallait se donner un mois de plus, se mettre autour d'une table et dire là on peut vraiment plus, alors trouvez une solution et une sortie honorable.

La soudaineté et la brutalité de l'expulsion hantent encore les esprits. Le souvenir de l'expérience est contrasté, "formidable » et " terrible », révoltante et désarmante. Les « travailleurs grévistes sans-papiers » s'en sont allés. Les associations et syndicats s'indignent de la façon dont l'accord a été rompu et demandent la réouverture des lieux. « Les travailleurs Sans-Papiers travaillent ici ! Vivent ici ! Payent des impôts et des taxes ici ! Ils doivent être régularisés immédiatement » : La CGT Culture s'inspire des mots d'ordre de 2008 « On bosse ici, on vit ici, on reste ici ! » pour rédiger son communiqué. Les journalistes et chercheurs commencent à écrire, les photographes se plongent dans leurs clichés et les illustrateurs préparent leurs planches. Tous sont animés d'un désir de conserver et transmettre.

\section{Patrimonialiser}

Le mouvement social suscite une dynamique patrimoniale, qui agit en simultané, voire en anticipation de son action. Elle émane des militants, des organisateurs et des « compagnons de route », artistes et intellectuels, voire de la CNHI.

Le syndicat CGT, qui structure le mouvement des « travailleurs grévistes sans-papiers », dispose d'un photographe officiel, qui couvre les différentes actions de ce collectif depuis plusieurs années. Son travail constitue autant de traces des événements et en cristallise la mémoire. Ce fonds est valorisé par le biais de la publication d'ouvrages à double visée artistique et militante (Rondeau et Esquerre 2009). Lors de l'occupation du Palais une exposition, produite par les militants, est montée dans le hall, avec le soutien de l'institution. Des photographies noir et blanc accompagnées de textes, tirées en grand format, offrent aux regards des visiteurs une 
galerie de portraits de travailleurs sans-papiers présents sur les lieux. Le choix des supports amovibles, prêts à être accrochés, laisse supposer la possible circulation de l'exposition. Cette forme de communication reprend des codes patrimoniaux identifiables de tous. Elle vise, à travers une forme légitime culturellement (relativement à un tract, par exemple), à informer les publics sur le sens de l'occupation de la CNHI, à les sensibiliser à la cause défendue et s'inscrit dans un dispositif de méthodes douces de conviction des opinions et de recherche de soutiens, éventuellement financiers.

Illustration 6

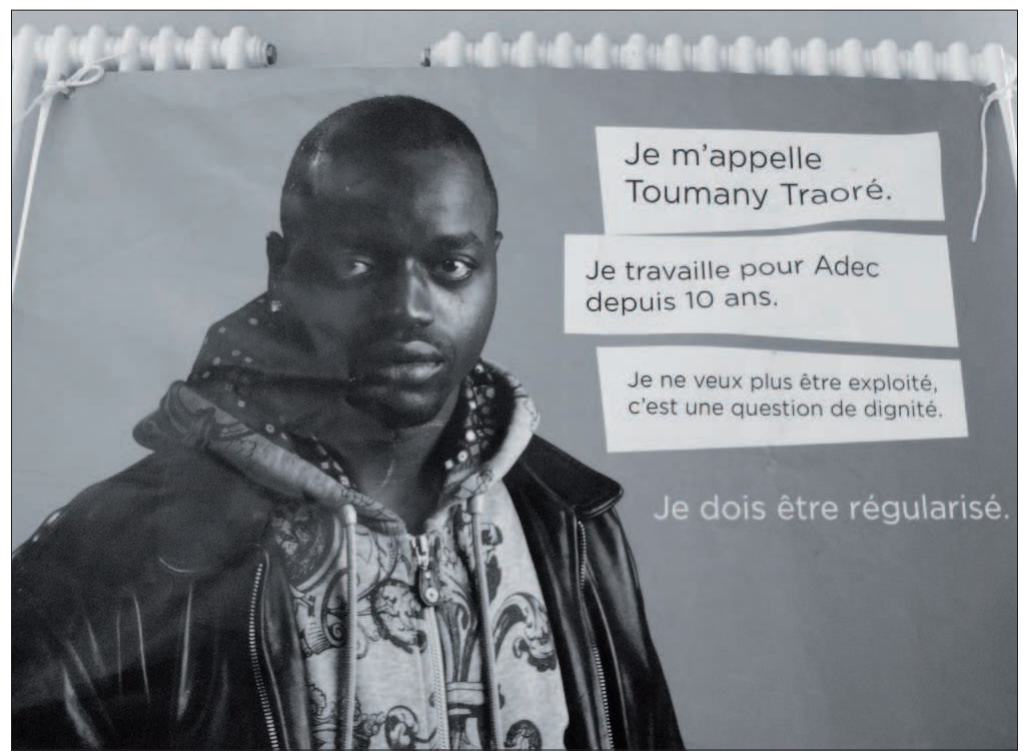

Détail de l'exposition militante des «travailleurs grévistes sans-papiers 》, Cité nationale de l'histoire de l'immigration. (C) Mélanie Roustan, janvier 2011.

Outre les images produites par les organisateurs, un certain nombre de productions sont issues d'artistes ou de scientifiques indépendants. Plusieurs photographes ont couvert l'occupation de la CNHI, par exemple Bruno Combes qui s'est rendu sur les lieux quotidiennement ${ }^{17}$. Quelques dessinateurs sont également venus croquer l'événement sur le vif, et poser leur œil sensible sur la rencontre pleine de symboles entre ces migrants en demande de reconnaissance administrative et le vénérable bâtiment porteur d'une mémoire collective partiellement refoulée. Certains d'entre eux suivent le mouvement depuis qu'il s'est initié, en 2009. Lionel Brouck, illustrateur pour des quotidiens et dessinateur engagé, a ainsi édité un recueil (2011), qu'il a promu, entre autres, par des expositions de ses planches en librairie ou en mairie d'arrondissement. Les profits réalisés viennent alimenter la caisse du collectif des « travailleurs grévistes sans-papiers ». La démarche est similaire pour la dessinatrice Laura Genz (Diallo et

17 Et dont nous publions ici quelques clichés. Nous tenons à le remercier pour sa confiance. 
al. 2010). Tous documentent le combat autant qu'ils s'en inspirent. La mise en images, qui se fait parfois mise en ouvrages, apparaît comme la forme de patrimonialisation privilégiée autour de ce mouvement social. Toutefois, la mise en textes n'est pas en reste, émanant largement mais non exclusivement du monde académique (Barron et al. 2011). Des universitaires constituent l'événement en objet d'étude ; bientôt, leurs articles et ouvrages paraîtront dans des éditions scientifiques. Le mouvement est par ailleurs relayé par des blogs, qui allient écrits et images dans des productions à tonalité plus personnelle, militante, artistique ou testimoniale.

En outre, le site internet de la CNHI et ses émanations sur les réseaux sociaux produisent un discours sur l'occupation, entre patrimonialisation et communication. Enfin, l'institution collecte la mémoire de l'événement, en même temps qu'elle le vit, suivant en cela les principes de sa mission : son objet de patrimonialisation «officiel » n'est-il pas celui de l'histoire et des cultures de l'immigration ? Elle récupère les affiches et les tracts ; plus tard, elle s'occupera des images produites par différents témoins et acquerra les publications ayant trait au mouvement. Une collection thématique est constituée, s'inscrivant dans la politique scientifique et culturelle de l'établissement. Le département iconographie de la médiathèque et le service de la conservation du musée s'engagent ainsi dans une démarche de rassemblement d'un fonds (plutôt documentaire ou plutôt artistique) autour de l'occupation des lieux par les « travailleurs grévistes sans-papiers ». Lors d'un entretien, un conservateur explique :

Nous avons commandé immédiatement un film bien précis, quelques témoignages. Nous avons sollicité beaucoup d'artistes avec lesquels nous travaillons : photographes, illustrateurs... Et nous avons proposé de faire entrer dans les collections six dessins qui montrent l'occupation du bâtiment. (...) Cela fait partie de l'histoire du bâtiment et peut-être un jour, cela servira s'il y a un espace historique au sein des expositions. (...) Notre vocation première n'est pas de rebondir sur l'actualité (...) mais cela nous a semblé essentiel de garder cela comme témoignage patrimonial pour l'avenir.

Les événements sont digérés, leurs traces patrimonialisées. Voyant dans le présent l'histoire en train de se faire, l'institution « s'auto-patrimonialise ».

\section{Conclusion}

La dynamique patrimoniale se fonde sur des régimes d'historicité où le présent passe de plus en plus rapidement (Hartog 2003). Des plus hautes institutions (Heinich 2009) aux initiatives individuelles les plus infimes (Rautenberg 2003), des forces sociales les plus traditionalistes aux plus contestataires, toutes tendent à produire - et consommer - le présent comme un temps révolu, à conserver plutôt qu'à détruire ou à renier. Sur le mode de l'accélération, le présent se vit comme un passé à venir, dont il faudra se souvenir. La rhétorique patrimoniale inscrit les choses dans une vision linéaire du temps. Le contemporain se voit redevable de son passé - un passé dont il faut assurer la sauvegarde (conservation). Cette dette nourrit un discours éthique, qui ménage connaissance (histoire), reconnaissance (mémoire) et neutralisation (patrimoine). 
La force du paradigme patrimonial au sein de nos sociétés apparaît. Il domine les autres lectures possibles du monde, plus engagées, voire plus polémiques - non seulement en les surplombant, mais en les absorbant, presque en les « cannibalisant» (Hainard et al. 2002). Comme le « nouvel esprit du capitalisme », prompt à " incorporer » les mouvements qui tentent de le remettre en cause, y compris la « critique artistique » (Boltanski et Chiapello 1999), la patrimonialisation digère sa propre contestation. Elle s'immisce dans toutes les sphères de l'activité, y compris militante, comme si la légitimité sociale ne pouvait venir que d'une reconnaissance culturelle. Appuyée sur les registres symboliquement dominants des arts, des lettres et des sciences (produire des images, des mots, créer), elle rend les messages plus audibles et leur assure une large diffusion. Mais elle transforme aussi le présent en une page à tourner. Qu'il s'agisse de contester le patrimoine ou de l'utiliser pour contester, la digestion patrimoniale simultanée neutralise les enjeux politiques. En fixant le mouvement social, en l'esthétisant ou en l'intellectualisant, ne stérilise-t-elle pas son action?

Pour les «travailleurs grévistes sans-papiers », la lutte n'est pas terminée, l'avenir reste à écrire. Du côté du Palais de la Porte Dorée, il semble impossible pour l'institution de s'affranchir du stigmate du musée des Colonies et de se démarquer d'une tutelle à un ministère controversé. La pérennité du message incarné par l'édifice dépasse les tentatives de réinterprétation qui en sont faites. Les approches populaires le rappellent à ses fonctions premières, à la fois de glorification des altérités françaises et d'incarnation d'une politique de l'État discriminatoire à leur égard. La digestion patrimoniale est douloureuse, tant elle peine à trouver un sens à son action, un ordre à sa réinterprétation. Car penser les liens entre présent et passé revient à aborder les relations entre l'ici et l'ailleurs de la citoyenneté française : ancrage territorial en extension ou en repli (empire colonial versus fermeture des frontières), figures évolutives de l'étranger et de son intégration à la nation, de l'indigène à l'immigré. Penser ces liens induit une réflexion - et un positionnement - politiques sur les façons d'envisager en France l'autre et le même (principe d'égalité versus concept d'identité).

\section{Références citées}

Bancel, Nicolas et Pascal Blanchard, 2007. «Incompatibilité : la CNHI dans le sanctuaire du colonialisme français », Hommes et Migrations 1267.

Barron, Pierre, Anne Bory, Lucie Tourette, Sébastien Chauvin et Nicolas Jounin. 2011. On bosse ici, on reste ici! La grève des sans-papiers : une aventure inédite. Paris : La Découverte.

Bensa, Alban et Eric Fassin, 2002. «Les sciences sociales face à l’événement », Terrain 38 : 5-20.

Blanc-Chaléard, Marie-Claude, 2006. «Une Cité nationale pour l'histoire de l'immigration. Genèse, enjeux, obstacles », Vingtième Siècle. Revue d'histoire 4 (92) : 131-140.

Boltanski, Luc et Eve Chiapello, 1999. Le nouvel esprit du capitalisme. Paris : Gallimard.

Brouck, Lionel, 2011. Voyage au bout de l'envers. Reportages dessinés. Auto-édition [vente en ligne : http://brouck.canalblog.com/].

Cohen, Anouk, 2007. «Quelles histoires pour un musée de l'Immigration à Paris ! », Ethnologie française « Mémoires plurielles, mémoires en conflit » 3 (37) : 401-408.

Collectif, 2007. Museum international : « Patrimoine des migrants », 233-234. 
Collectif, 2005. Diasporas : «Migrations en mémoire », 6.

Collectif, 2004. Hommes et Migrations : «Vers un lieu de mémoire de l'immigration », 1247.

Diallo, Mamoudou, Fofana Vazoumada et Laura Genz, 2010. Hier colonisés, aujourd'hui exploités, demain régularisés. Les journées de la coordination 75 des Sans-Papiers. Paris : Fage éditions.

Eidelman, Jacqueline, Anne Monjaret, Mélanie Roustan (textes) Bernard Plossu (photographies), 2002. MAAO Mémoires. Paris : Marval.

El Yazami, Driss et Laurent Schwartz, 2001. Rapport pour la création d'un Centre national de l'histoire et des cultures de l'immigration. Dactylographié.

Fabre, Daniel, 2009. « Habiter les monuments », in Daniel Fabre et Anna Iuso (dir.), Les monuments sont habités, 17-52. Paris : Editions de la Maison des sciences de l'homme.

Green, Nancy, 2011. "Construire une collection, représenter l'immigration : la Cité nationale de l'Histoire de l'immigration », Hermès : « Les musées au prisme de la communication » $61: 131$ 137.

Grognet, Fabrice, 2009. Le concept de musée. La patrimonialisation de la culture des " autres ». D'une rive à l'autre, du Trocadéro à Branly : histoire de métamorphoses, Thèse de doctorat en ethnologie. Paris : EHESS.

Hainard, Jacques, Roland Kaehr et Marc-Olivier Gonseth (éds), 2002. Le musée cannibale. Neuchâtel : Musée d'ethnographie.

Hartog, François, 2003. Régimes d'historicité. Présentisme et expériences du temps. Paris : Seuil.

Heinich, Nathalie, 2009. La fabrique du patrimoine. De la cathédrale à la petite cuillère. Paris : Éditions de la Maison des sciences de l'homme.

Linhart, Danièle, Barbara Rist et Estelle Durand, 2004 [2002]. Perte d'emploi, perte de soi, Ramonville Saint-Agne : Erès.

Monjaret, Anne, 2005. «Quand les lieux de travail ferment », Ethnologie française « Fermetures : crises et reprises » $4: 581-592$.

-, 2008. « La fête de fermeture de trois hôpitaux parisiens, ou comment se marque, se ritualise et se fabrique l'événement ", in Michel Balard et Noëlle Gérôme (éds), Revue de la Fédération des sociétés historiques et archéologiques de Paris et de l'Ile-de-France « Les fêtes et leur histoire en Ile-de-France- Paris et Ile-de-France Mémoires » 59 : 333-356.

Monjaret, Anne, Mélanie Roustan et Jacqueline Eidelman, 2005. «Fin du Musée National des Arts d'Afrique et d'Océanie : un patrimoine revisité ", Ethnologie française " Fermetures, crises et reprises » $4: 605-616$.

Rautenberg, Michel, 2003. La rupture patrimoniale. Paris : À la Croisée.

Rondeau, Bernard (photographies) et Marion Esquerre (textes), 2009. Des hommes libres. Une histoire de la grève des travailleurs sans-papiers. Paris : Le Cherche Midi.

Toubon, Jacques, 2004. Mission de préfiguration du centre de ressources et de mémoire de l'immigration. Rapport au premier ministre. Paris : La Documentation française. 